\title{
Using POI functionality and accessibility levels for delivering personalized tourism recommendations
}

\author{
Filipe Santos ${ }^{\mathrm{a}, *}$, Ana Almeida ${ }^{\mathrm{a}}$, Constantino Martins ${ }^{\mathrm{a}}$, Ramiro Gonçalves ${ }^{\mathrm{b}}$, José Martins ${ }^{\mathrm{b}}$ \\ a Institute of Engineering - Polytechnic of Porto, Porto, Portugal \\ ${ }^{\mathrm{b}}$ INESC TEC - Universidade de Trás-os-Montes e Alto Douro, Vila Real, Portugal
}

\section{A R T I C L E I N F O}

\section{Article history:}

Received 10 June 2017

Accepted 14 August 2017

Available online $\mathrm{xxxx}$

\section{Keywords:}

Tourism

Recommendation system

User profiles

Point-of-interest

Emotions

Tags

\begin{abstract}
A B S T R A C T
The aim of this paper is to propose a recommendation system that considers user's functionality levels regarding physical or psychological limitations. This paper describes a set of models and algorithms used under a tourism recommendation system based in users and points-of-interest (POI) profiles. Also, this proposal considers a different manner to classify POI including their accessibility levels, mapped with similar physical and psychological issues. In this study, based on the Design Science Research methodology, an architecture is proposed and a touristic recommendation system prototype where users are modelled with new types of information in addition to traditional approaches such as their levels of functionality regarding a set of physical and intellectual issues, is also presented. POIs are also modelled with the same information structure and maintain knowledge on their limitations against some health conditions.
\end{abstract}

(c) 2017 Elsevier Ltd. All rights reserved.

\section{Introduction}

Tourism is, in a global perspective, one of the fastest growing areas in the economy (Martins et al., 2017), but the appearance of new offers in different geographies with new attractions, creates important challenges to traditional destinations that need to evolve and turn themselves the best option for travellers.

According to the Secretariat for the "Convention on the Rights of Persons with Disabilities" (UN, 2016), urbanization is currently one of the most important global trends of the 21 st century. It has the potential to be the engine for achieving sustainable and inclusive development for all, if urban environments, infrastructures, facilities and services are also planned and built for all.

Limitations or impairments are characterized as a significant biomedical change in the structure or physical and psychological functions of the human body, compared to a normal pattern. However, the occurrence of these limitations should not immediately determine a disability, it may be possible to find valid alternatives to became possible for an individual to enjoy a certain experience, regardless of his physical or mental state (Santos, de Almeida, Martins, de Oliveira, \& Gonçalves, 2017).

A disability can be characterized like the result of adding a deficiency and a certain environment whose characteristics provide the individual conditioning (Salsgiver, 2015).

\footnotetext{
* Corresponding author at: Institute of Engineering - Polytechnic of Porto, Compute Science Department, GECAD Knowledge and Decision Support Research Group, Portugal. E-mail addresses: jpe@isep.ipp.pt (F. Santos), amn@isep.ipp.pt (A. Almeida), acm@isep.ipp.pt (C. Martins), ramiro@utad.pt (R. Gonçalves), jmartins@utad.pt (J. Martins).
}

For the $15 \%$ of the world's population who live with a disability, available evidence reveals a widespread lack of accessibility associated with environments, infrastructures, public buildings and services, and a significant difficulty for those citizens to access information and communications (UN, 2016). These accessibility limitations contribute greatly to the disadvantage and marginalization faced by persons with disabilities, leading to disproportionate rates of deprivation and exclusion.

The abovementioned evidence is also observed in the tourism domain, where accessibility conditions not only improve the disabled tourists experience enjoyability, but also allow for all tourism experiences to be more feasible for all, especially for elderly people and a wider-angle of the population. Scientific studies have shown that the number of tourists with disabilities travelling to other countries for tourism purposes is only a little below average when compared with non-disabled tourists (Neumann, 2002). Therefore, it is recognizable, that people with disabilities do travel on tourism and represent, under this perspective, an important target group.

Each tourist is different and has a unique "luggage" of experiences, motivations and solicitations (Condratov, 2013). The contemporaneous traveler is less and less disposed to wait for obtaining information on the visited destinations, thus the key of success within tourism organizations consists in the fast identification of customers' necessities and saluting the potential customers with offers as varied as possible, personalized and updated to current tendencies (Dimitrios Buhalis, 2008).

One of the most effective ways to respond to the abovementioned challenges is to improve knowledge on tourists, tracing their individual profiles with the most appropriate and distinct information, like physical and psychological issues, personal tastes and similarity with others. 
User modelling, as a unique and individual process, is usually poor and clearly rudimentary either in the methods or in the scope, because a significant part of the available data is not treated and used in order to allow for a more correct knowledge of each entity. Traditional user modelling approaches also tend to devote low attention to key issues such as physical and/or mental limitations of each user, which often are inhibiting factors for the activities that each one can carry out.

Despite the technology evolution related with users' profiles modelling (it has become more complex and intelligent, due to use of recent technological evolutions), the basic concepts, ideas and problems that supported the appearance of this research area are almost the same: the identification of user needs, desires, personalities and, most important, objectives. For example, in a research work related with education processes (Faria, Almeida, Martins, Gonçalves, \& Figueiredo, 2015; Martins et al., 2013) authors model students as individuals that differ in their social, physical, psychological, emotional and also ethnic characteristics in order to obtain better student's results.

User modelling implementation is normally performed recurring to two sets of techniques: a knowledge-based technique or a behavioural technique (Kobsa, 2001; Martins, Faria, De Carvalho, \& Carrapatoso, 2008). Knowledge-based approach is normally the result of information gathered using forms, queries and other user studies, with the purpose of producing a set of heuristics. Behavioural adaptation is related with user monitoring during his daily tasks and activities.

For the purpose of our research we propose a knowledge-based approach regarding the usage of information collected through forms. The main focus is the consideration of distinct user related information like the relation between a user and a set of stereotypes defined by “Turismo de Portugal" (business, nature, sun and sea, etc.), user's emotional reactions to various stimulus, tags related with each user and user's functionality levels regarding physical and psychological issues.

Tourism recommendation systems can play a key role in improving user's experiences and consequently the perception that they acquire of a certain location. The results can be based in a merge between a knowledge on the user and a detailed characterization of each Point of Interest (POI), hence allowing the referred systems to make suggestions capable of avoiding or at least minimize the tourist disabilities or impairments impact during their tourism experience.

According to Hu et al. (2015), a Point of interest (POI) represents individual locations (e.g., a restaurant or a landmark) which are of interest to people (McKenzie, Janowicz, Gao, Yang, \& Hu, 2014; Yoshida, Song, \& Raghavan, 2010).

A recommendation system can be defined as a collection of different techniques used to filter and organize different items with the objective of selecting either the best ones or the most suitable ones for the user profile (Lucas, da Silva Coelho, García, de Almeida Figueiredo, \& Martins, 2011; Luz, Anacleto, Martins, Almeida, \& Lucas, 2013; Porter, 2006). Although the most common scenario is when the system has to choose the best items from a certain group which, otherwise, (without the filtering) would be randomly selected. There are other more important cases where certain items or types of items just can't be shown to the user at a given moment, for example, due to handicap issues. A complete recommendation system should therefore, be prepared to handle both types of situations.

Points of interest (POI) are inextricably linked to modern recommendation systems, location-based social networks, transportation studies, tourism systems, urban planning, and so forth (McKenzie, Janowicz, Gao, \& Gong, 2015). In terms of their computational representation, a POI can be described and categorized in many different ways. Typical approaches are either based on features or functionality. In this work are considered both when defining a POI profile.

In this research is considered that the best manner to accomplish user's objectives and preferences respecting his stereotype relation accuracy and functionality levels, according to the POI profile.

Stereotype profile is related to the aim of a tour, for example, nature, business, residential, etc. Functionality levels are related with user's abilities like locomotion, vision and psychological/intellectual issues. The accessibility levels defined for each POI can be described as part of the POI profile, this is also a key difference in this work when compared with others. These two research areas, USER/POI modelling and recommendation systems, are the most important part of this work, in addition to a tool set used to collect and process important data related with a specific user, his preferences (stereotype, tags, etc.) and physical and psychological limitations.

The main goal of this research is the development a recommendation system where users are modelled considering, in addition to traditional approaches, new types of information such as their levels of functionality regarding a set of physical and cognitive issues. POI's are also modelled with the same information structure particularly in the case of physical and psychological limitations, thereby allowing the definition of their accessibility level.

This research can be very relevant for the tourism recommendation systems research field and any other areas where more accurate individual user knowledge is the key factor to obtain better results. The better fulfilment of individual user's objectives in coexistence with the respect for their own physical and psychological limitations is probably one of most important objectives for this research work.

\section{Tourism recommendation systems}

When referring to tourism domain, there are a lot of recommendation systems, although with different purposes. Some these systems only focus on some aspect of the holidays. For example, Personal Travel Assistant (PTA) (Coyle \& Cunningham, 2003) is used for reserving and selling flights. Entreé uses domain knowledge about restaurants, foods and cuisines to recommend restaurants to users (Burke, 2000). Similarly, (Tung \& Soo, 2004) propose CAPA, a personalized restaurant recommendation software agent that runs on mobile devices. In a quite different domain, (Huang \& Bian, 2009) have also developed a recommendation system for suggesting specific tourist attractions over the Internet.

Mobile tour guides are the result of years of research in the areas of recommendations, ambient intelligence and pervasive computing. Mobile recommendation systems based on profiles have the potential to substantially enrich tourist experiences. As their handling marks a big challenge for ordinary users, its acceptance can only be evaluated when utilized by the intended user group itself - real tourists.

There are systems that only display information about sights, like MultiMundus (Tusch, 2007) which primary goal is to provide multimedia information of a sight to the tourist on his personal mobile device. With this content, it can provide moderated audio guides for travel groups, automatic detection of the tourist physical position on the map and presentation of the sight closest to him.

TIP (Hinze \& Buchanan, 2005) and Heracles (Ambite, Knoblock, Muslea, \& Minton, 2003) provides recommendation services through mobile devices for tourism. These services implement hybrid algorithms to calculate tourist preferences, using the defined tourist profile and location data (location-aware).

Proximo (Parle \& Quigley, 2006) is a location-aware mobile and recommendation system that fits the pure paradigm approach. It guides users through tours within buildings using Java and Bluetooth technologies. The mobile device also tracks the user location and builds a context, providing the system with important information. The user position is taken by "sniffing out" the fixed Bluetooth devices or low-cost beacons deployed in the area of use. Proximo pure collaborative recommendation system relies on its user's item ratings to provide recommendations.

In GeoNotes (Espinoza et al., 2001) system tries to blur the boundary between physical and digital space (ubiquitous computing and augmented reality). At the same time, it strives to socially enhance digital space (collaborative filtering, social navigation, etc.) by allowing users to participate in the creation of the information space. GeoNotes is a location-based information system that allows the user to access information in relation to the user's position in geographical space. 
The tourism-oriented mobile GIS (geographic information system) application MacauMap (Biuk-Aghai, 2003) designed for the city of Macau allows map navigation while displaying the user current location. It also provides information about the public bus network and bus guides for calculating optimal bus routes. It also provides sightseeing guides with information about museums, churches, temples, hotels, restaurants and other places of interest, along with their location on the map.

EtPlanner is a mobile planning assistant (Höpken et al., 2006) that allows the creation of personalized tourism stays. Using a mobile device (e.g. a PDA or mobile phone) the costumer's stay is intelligently planned. This way the user can be assisted before, during and after his journey.

The personal mobile assistant mobiDENK (Krosche, Baldzer, \& Boll, 2004) has been developed for a tour to the Herrenhausen Gardens in Hanover and includes POI on which historical information and images of the most significant features are presented on a PDA. It focuses on drawing the user's attention to historic sites and provides locationbased multimedia information at different sightseeing spots while displaying the person's current location on a map.

Cyberguide system (Abowd et al., 1997) was developed at the Georgia Institute of Technology (GIT), Atlanta, USA. It is based on the ubiquitous computing concept and focuses on mobile context-aware tour guide. The system was designed to assist a visitor in a tour to the GIT, and helps the user obtaining information about the demos in display. Knowledge of the user's current location, as well as a history of past locations are used to provide more of the kind of services that we come to expect from a real tour guide.

CATIS (Pashtan, Blattler, Heusser, \& Scheuermann, 2003) is a contextaware tourist information system with a Web service-based architecture. The context elements considered to this project are location, time of day, speed, direction of travel and personal preferences. This system will provide the user with relevant information according to his location and the current time.
Deep Map application (Malaka \& Zipf, 2000) realizes the vision of a future tourist guidance system that works as a mobile guide and as a web-based planning tool. It is a mobile system that aids tourists with navigating through the city of Heidelberg by generating personal guided tours. Such a tour shall consider personal interests and needs, social and cultural backgrounds (e.g. age, education and gender), type of transportation (e.g. car, foot, bike or wheelchair) and other circumstances from season, weather and traffic conditions, to time and financial resources.

In Tousplan project (Almeida, Coelho, \& Martins, 2010; Coelho, Figueiredo, \& Martins, 2009) a Tours Planning Support System (TOURS PLAN) is proposed which intends to help tourists in finding a personalized tour plan allowing them to use their time efficiently and promote the culture and national tourism. Hence, this research focuses on tour planning support, aiming to at define and adapt a visit plan combining, in a tour, the most adequate tourism products, namely interesting places to visit, attractions, restaurants and accommodation, according to tourist's specific profile (which includes interests, personal values, wishes, constraints and disabilities) and available transportation modes between the selected products. Functioning schedules are considered as well as transportation schedules. This project tries to efficiently address the core of the tour planning process. Hence, it defines an optimization model that clearly represents the described tour-planning problem and designs a heuristic algorithm that effectively tackles that problem.

ITravel application (Yang \& Hwang, 2013) is based in ratings provided by other tourists with similar interests. The approach used employs mobile peer-to-peer communications for exchanging ratings via their mobile devices. Data exchange is based in wireless RF-communication technologies present in mobile devices that allow users to effectively share their ratings toward visited attractions.

3D-GIS Hybrid is a context-aware mobile recommendation system whose goals are Ubiquity (users may use the system wherever they like using the mobile platform), Location-awareness (recommendations

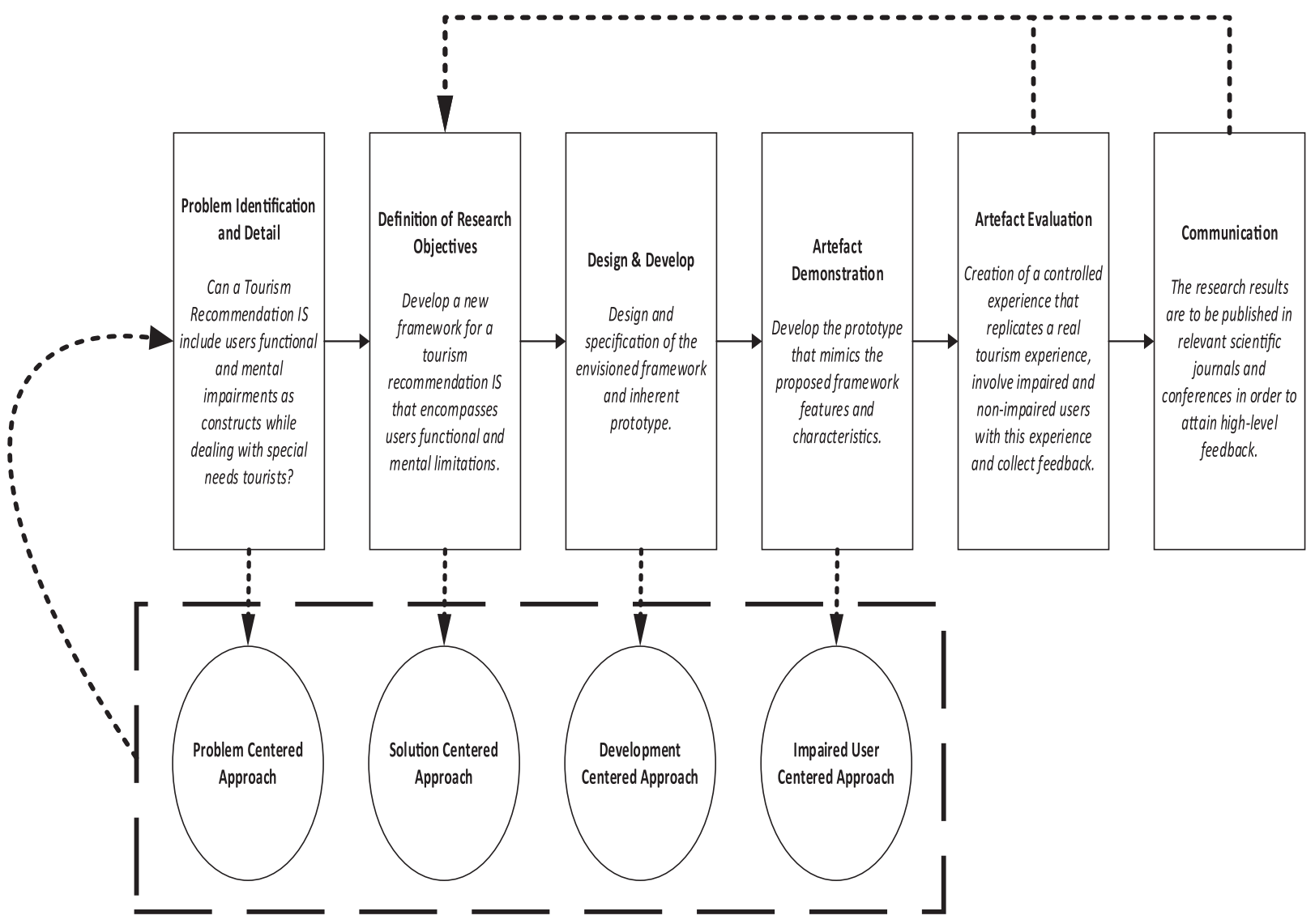

Fig. 1. Representation of the project research methodology. Drawn from Peffers et al. (2007) Design Science Research methodology. 
provided are adapted to the user's current location) and 3D-interface (it includes a 3D solution with innovative features as 3D geovisualization, location, etc.) (Noguera, Barranco, Segura, \& Martínez, 2012).

POST-VIA 360 (Colomo-Palacios, García-Peñalvo, Stantchev, \& Misra, 2017), is a platform devoted to support the whole life-cycle of tourism loyalty after the first visit that is designed to collect data from the initial visit by means of pervasive approaches. This data is used to produce accurate after visit data and, once returned, is able to offer relevant recommendations based on positioning and bio-inspired recommendation systems. The validation is based in a case study comparing recommendations from the application and a group of experts.

The TRSO project (Chu, Wang, Zheng, Wang, \& Tan, 2016) describes in detail a tourism recommendation system based on ontologies. Initially, the system uses association rules to relate users from an extended group. From this moment, the system will have in its database two categories of users, related and unrelated. For the related users, a collaborative recommendation algorithm is proposed. For unrelated users, a different collaborative recommendation mechanism is used that integrates information from the ontology about tourist attractiveness.

The web application ETourism 2.0 (Ibáñez, Sebastia, \& Onaindia, 2016) was developed to allow the generation of customized tourist tours recommendations in the city of Valencia. This application takes into account a set of user preferences to define the tourist style of each. This approach considers important to relate the duration of the visit to a given point with the interest of a user to carry out the same visit, allowing to consider the preferences of users in the configuration of their agenda in terms of the number of places to visit and the total duration of the tour.

This work proposed in this paper differs from the ones hereby described because it's main focus is user functionality/POI accessibility model in order to allow at the same time respect user's physical and psychological limitations and do not augment user's exclusion in terms of touristic experiences.

\section{Methodology}

When undergoing a research project one of the most important assets is the research methodology that supports it. A well thought and specified methodology allows for a validation of the research activities and by inherence of its results, hence valuing the research results and conclusions. This section provides for a brief characterization of the methodology used to support the present research project.

\subsection{Methodological approach characterization}

On their research, Gonçalves et al. (2015) argue that the use of information and communications technologies are optimal triggers for the development and management of user related information systems whose goal should be the overall improvement of users life.

As previously mentioned, existing tourism related recommendation information systems (RIS) are typically supported on a generalized set of user information that tends to ignore his specificities and provide for suggestions considered inadequate (Rivas-Costa et al., 2014).

On present research project, a choice was made to use Design Science (DS) Research methodology as the theoretical and guidance support that would allow to reach a successful outcome. When analysing the existing literature on the use of DS, one can almost immediately perceive that it is widely used throughout the information systems field (Gleasure \& Grace, 2016; Gregor \& Hevner, 2013). DS can be considered a pattern aimed at the specification and development of information systems and technologies artefacts whose goal is addressing (and solving) existing problems (Peffers, Tuunanen, Rothenberger, \& Chatterjee, 2007). Performing a DS research in the context of IST implies following a set of existing principals and guidelines whose focus is on the achievement of and artefact that not only solves an existing issue but also becomes important for both the organization and to the artefact users (Wieringa, 2014).

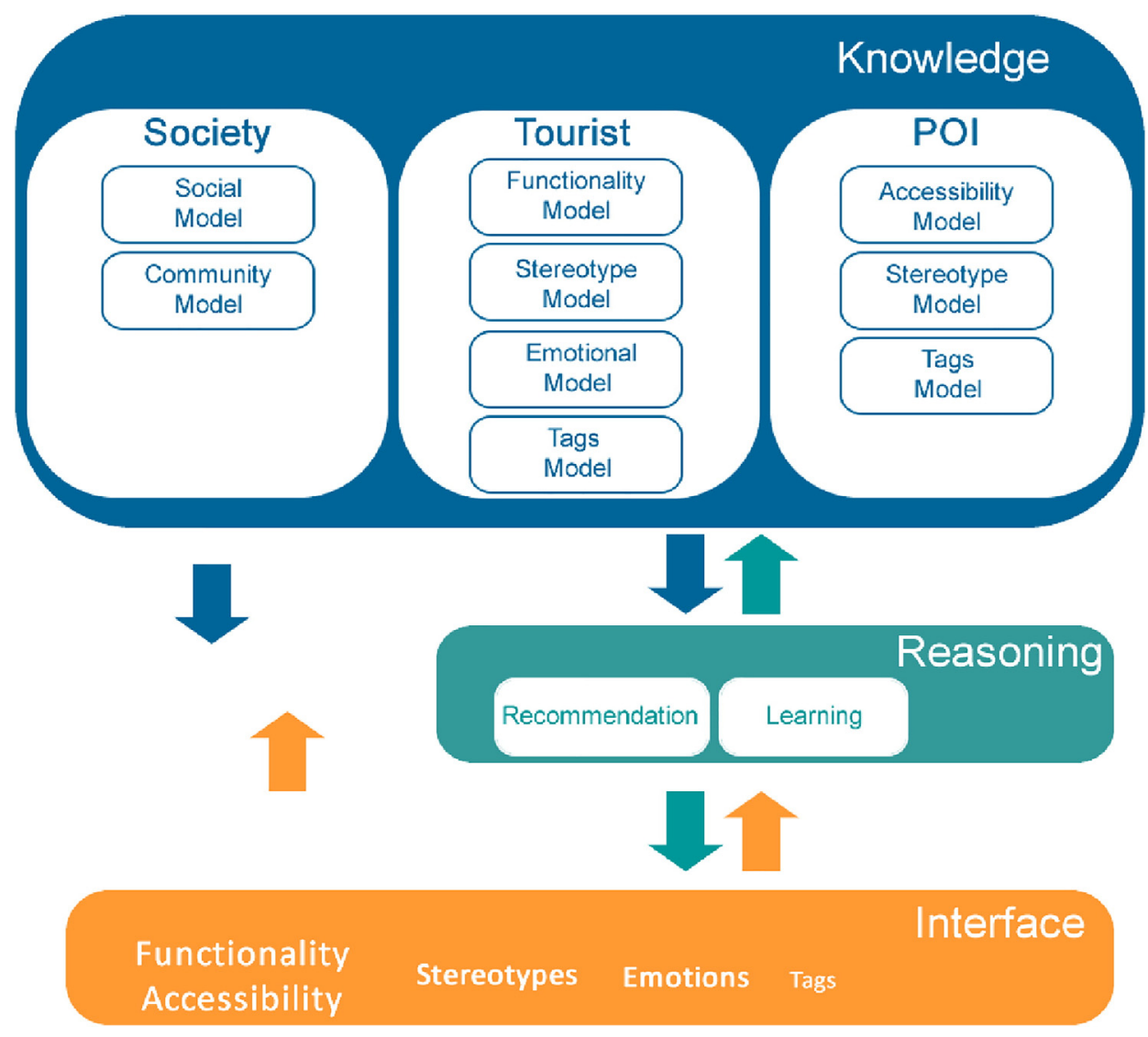

Fig. 2. POI recommendation system proposed architecture. 
For the scope of this manuscript, the developed artefact was a new framework that combines both a tourism related recommendation IS and a more advanced characterization of its user where his physical and mental pathologies or limitations are included, and the inherent prototype (Michopoulou \& Buhalis, 2013). This combination would, in theory, provide for proper recommendations, so important when perceiving a disabled or impaired tourist in an unknown touristic location.

Given the solution oriented position associated with DS methodology, this research project focused on initially reaching a comfortable level of awareness on the issues associated with the identified problem, thus allowing for in a very early stage to acknowledge that the most relevant concern relied on properly specifying tourism points-of-interest in order for them to encompass characteristics that relate with individuals physical and mental impairments (Kabassi, 2010).

The conceptualization of DS research indicates that the artefact validation/evaluation can be made through qualitative on empirical tests. With this in mind, for the scope of our research a decision was made to collect qualitative feedback from the prototype users and, in a cyclical manner, to re-feed the framework (and inherent prototype) with the collected knowledge, in order for them to reach the maturity level compliant with the identified needs. The collection of user feedback has been made by using a case study where a real experience, from the Portuguese city of Oporto, was artificially reproduced in a controlled environment and both disabled and non-disabled users were able to experiment the produced artefact and deliver their feedback.

\subsection{Research project rollout}

Drawing on DS methodology conceptualization and on Peffers et al. (2007) schematization of the methodology stages and activities, the present research project has been divided in several stages whose relation would create the conditions for reaching a successful set of results.

As one can perceive from Fig. 1, the present research project has been performed as a cyclic sequence of several stages. On the initial stage, the research team aimed at performing a comprehensive analysis to existing literature focused on recommendation information systems and its application to tourism and also to the specification and characterization of touristic points of interest by using (physical and mental impairments specific tags). This first effort allowed to reach a detailed awareness on the issues concerning the use of existing recommendation

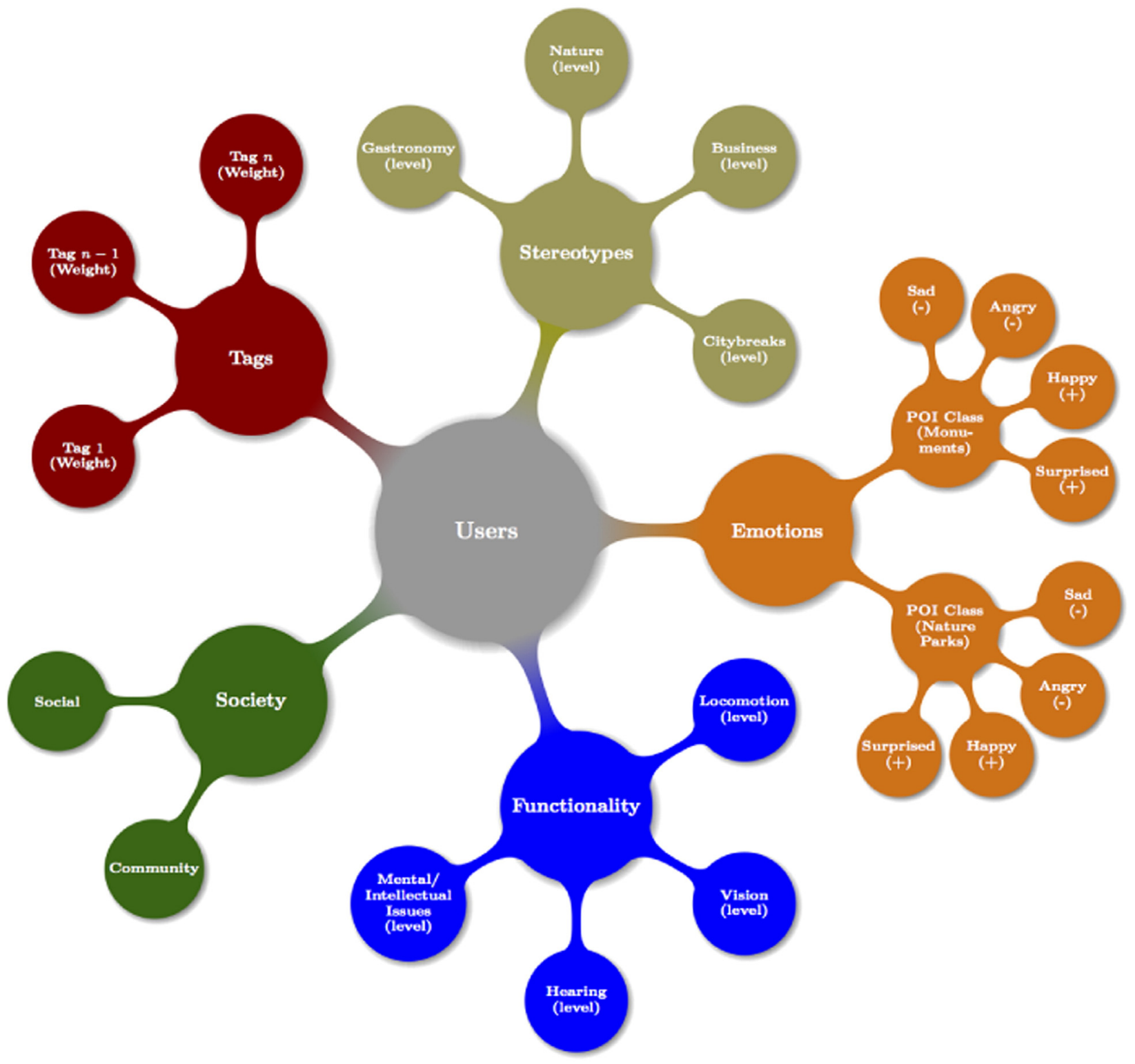

Fig. 3. User/tourist knowledge representation. 
IS by impaired or disabled users/tourists. By acknowledging this knowledge gap, a research objective has been outlined as the achievement of a new RIS framework that allows to characterize touristic points of interest (POI) with features such as the physical and mental characteristics that a user must have to be able to reach, or fully enjoy, the referred POI.

By merging the literature-based knowledge with the proposed research objective, a task directed at specifying and developing the research artefact was planned and executed. Given the scope of the artefact it was also necessary to develop a prototype that allowed for testing the achieved creation. This prototype virtually mimicked a real Oporto tourism experience in all its spectrum, except for the environment, given that our experiment was designed to have a controlled environment were all variables were accountable. Consequently, an experiment with users was drawn in order to validate the proposed framework and collect real feedback on its strength.

\section{Proposal}

This proposal intends to improve touristic experiences for tourists suffering from some physical or intellectual limitation. This is achieved recurring to the definition of functionality/accessibility levels that will allow for the creation of personalized POIs recommendation. From our perspective, it is only possible to detain a relevant knowledge on the tourists (and consequently provide for an accurate POI recommendation) if one understands the tourist three different contexts: a) the society context; b) the tourist context; and c) the POI context.

In order to address all the above-mentioned issues and goals, an architecture, divided in 3 layers (knowledge, reasoning and the interface), was proposed (Fig. 2).

\subsection{Knowledge layer}

This layer holds the knowledge regarding the different representation models considered for users/tourists (Fig. 2) namely: functionality, stereotypes, emotions, tags, society model and also the models used to represent POIs (accessibility, stereotype and tag models).

In Fig. 3 we present the models defined to characterize each POI. These models alongside with the knowledge that each one stores about the two main entities considered in this work (users and POI), are used by a set of algorithms, described in the reasoning layer, to produce accurate touristic recommendations according to both the users and the POI needs and characteristics.

\subsection{Reasoning layer}

The recommendation module can be described as a hybrid recommendation system given each of the subcomponents presented in Fig. 4 (the order in the figure is directly related with each component relevancy in the overall module) are based in different techniques used to obtain the basic knowledge to produce results.

The final recommendation plan is created applying RA1 algorithm that compiles the result of each local recommendation (stereotypes (genproresults), emotional states (emotional_results), tags (tags_results), society (society_results)) using a weighted sum (each model has a different weight in the overall recommendation process related with each one defined importance) of each POI value obtained in the local models. These local models base their results in the knowledge acquired and retained in the knowledge layer about each user and each POI.

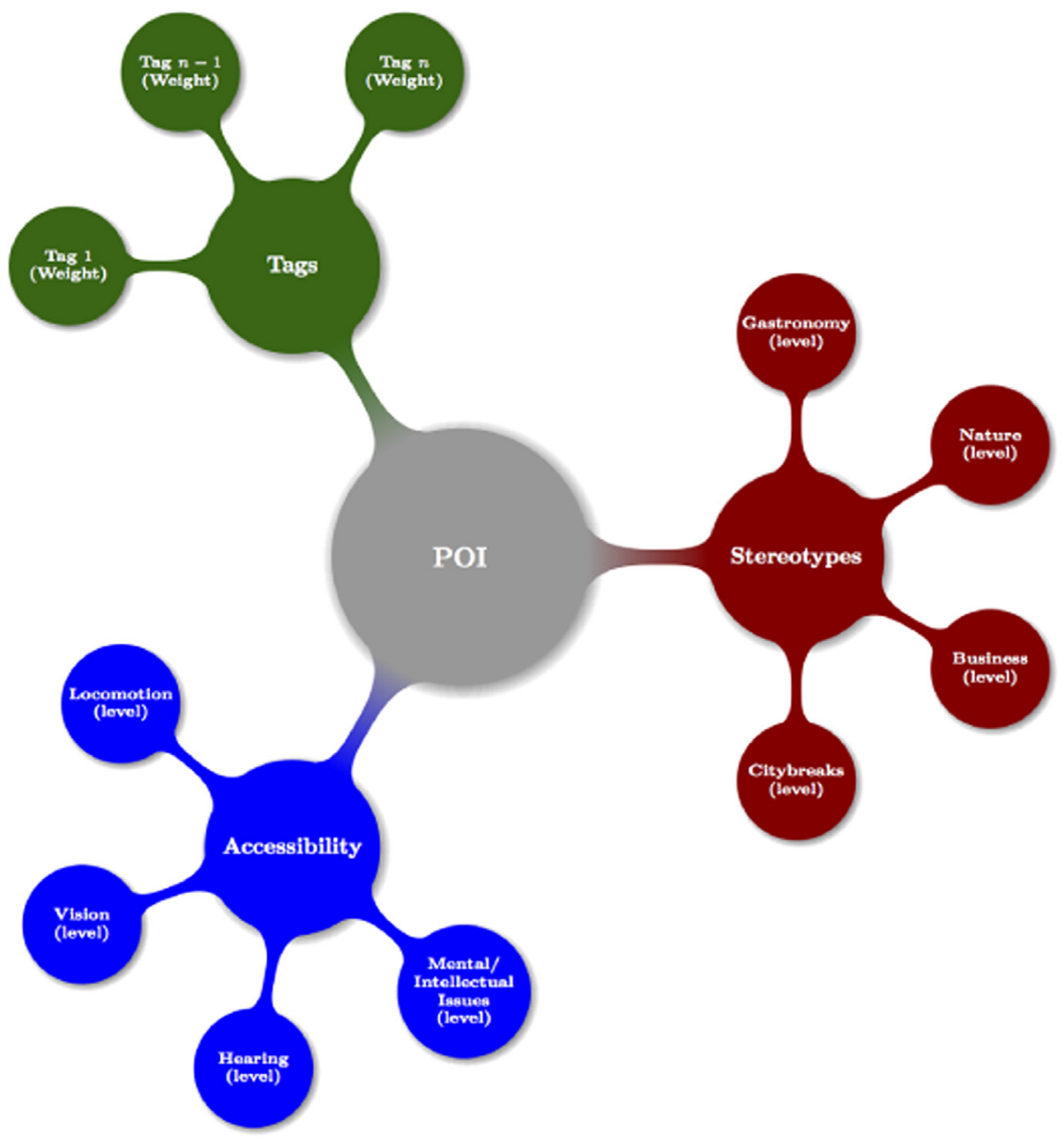

Fig. 4. Hybrid recommendation system components. 
This resultant recommendation is further refined according to functionality/accessibility model results (functionalaccessib_results) in order to guarantee that user's physical and psychological limitations are respected and the best touristic experience possible is suggested. This refining process is made calculating POI position indexes average considering the above referred resultant arrays (functionality/accessibility local results and stereotypes, emotional states, tags and society weighted calculated results).

In this process, all POI's are considered and none of them are excluded from the possible recommendation results in the beginning, unless if a POI is part of a class that user specifically denies (if a user denies a class, all POI's that composes that class are not considered to the recommendation).

The hybrid tourism recommendation algorithm is shown below.

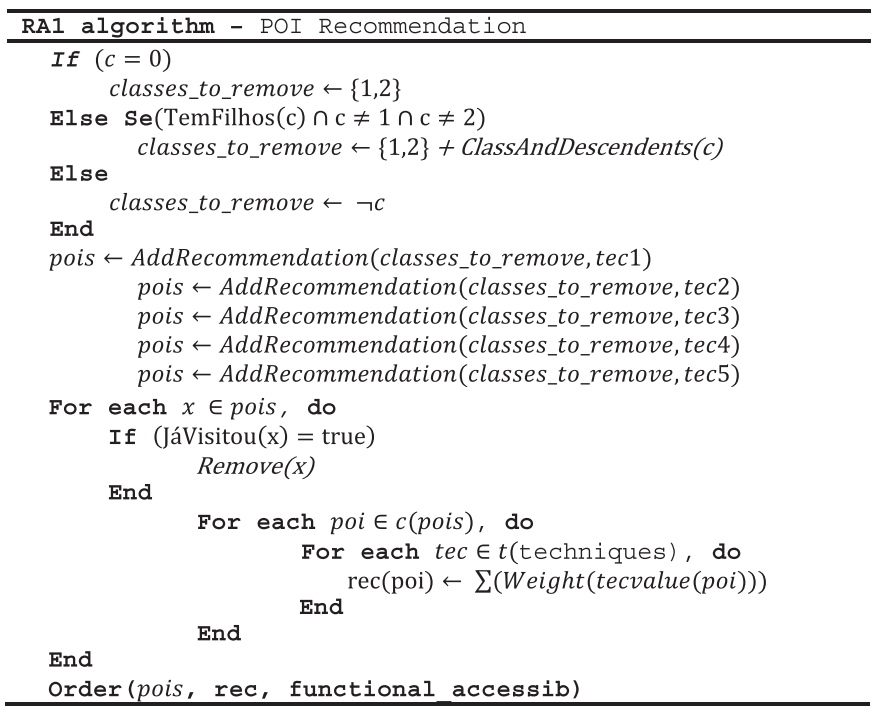

\subsubsection{Functionality/accessibility recommendation model}

The recommendation based in the functionality/accessibility model is based in the relation between the user's functionality level regarding a specific physical or psychological deficiency (for instance locomotion, vision, hearing or intellectual pathologies) and the accessibility levels obtained by POI caracterization and evaluation process accordingly the same refered physical and psychological constraints. This model proposed is an evolution of the the proposal made by Santos et al. in (Santos et al., 2017).

RA2 algoritm described below associates user's functionality levels with $\mathrm{POI}$ accessibility levels regarding the same pathology, which allows the creation of recommendation results that respect both user's limitations and POI characteristics.

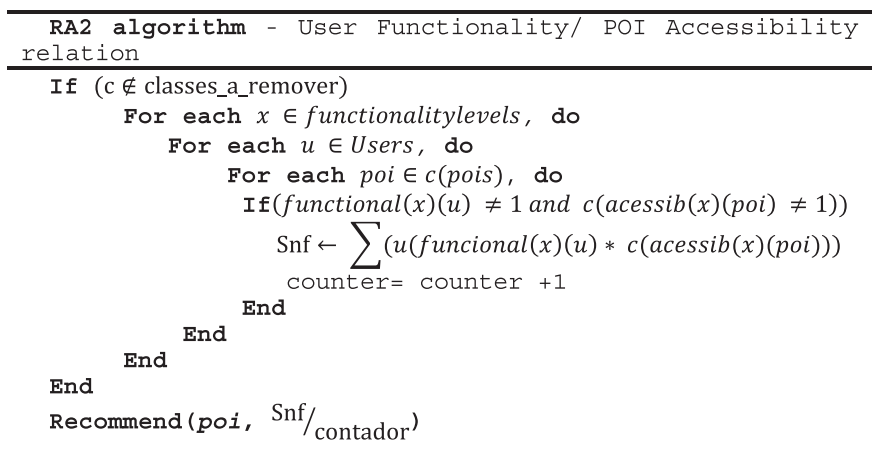

During the process of algorithm execution, if the functionality (user) or the accessibility (POI) levels regarding some limitation are equal to the maximum value in the considered scale $(0-1)$ this limitation is dismissed in this classification. In this case doesn't exist any obstacule to persons regarding a specific limitation or deficiency (either the user has full functionality or the POI does not create any obstacule).

\subsubsection{Stereotypes based recommendation model}

The recommendation process drawn on the comparison between the user's characteristics and a set of stereotypes (previously defined by Turismo de Portugal (2006)), can be classified as a behavioural-based technique.

In this technique, POI's are ordered and selected accordingly their relation with the stereotypes considered. Notice that stereotypes were used to characterize users considering their preferences and characteristics. This method has a weight of 4 in the overall recommendation process because it performs an important role in the detection of user's preferences and characteristics.

RA3 algorithm, described below is used to produce touristic recommendations based of the relation between POI and users profile.

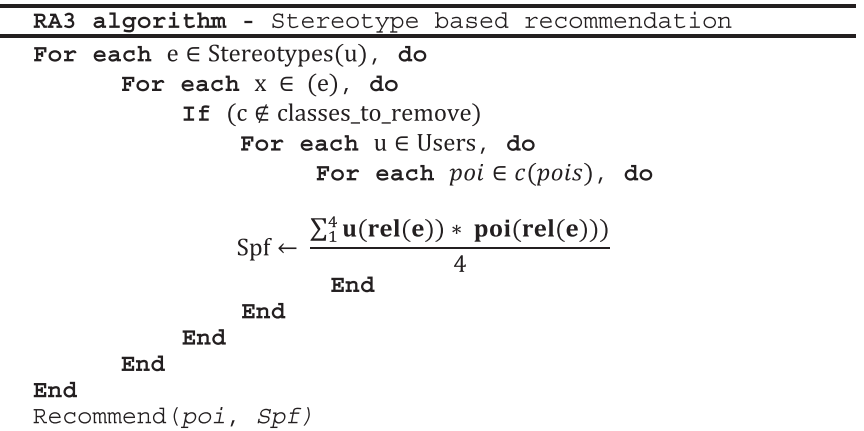

\subsubsection{Emotions based recommendation model}

This recommendation process is based in the emotional reaction that the system has detected when a set of images representing POI classes where shown to users.

This reaction (emotional state) allows to classify a POI using the emotional obtained result for his class.

RA4 algorithm, described below is used to produce touristic recommendations based of the relation between POI and users profile.

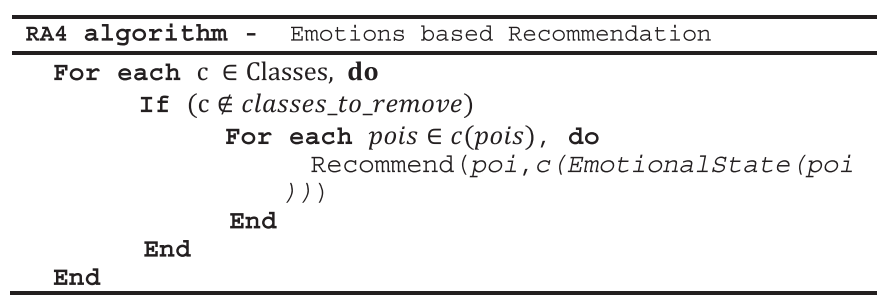

\subsubsection{Tag based recommendation model}

Tags are part of knowledge representation mechanism used to collect user's preferences. This method suffers from the uncertainty related with the almost infinite possibilities, which can produce recommendation results that are really diversified (language ambiguities can create uncertainty in the process). This method can be classified as a content filtering technique.

This technique can be an interesting approach to considerer because with the constant growth, in the recent years, of social networks presence, the data available to process and then extract new knowledge 
is exponentially growing. This can help systems to detect and represent users' preferences more accurately and with less effort. The importance of tags was studied during the research project and has been previously published (Santos \& Almeida, 2011; Santos \& Almeida, 2013).

In this method, tags have a specific weight calculated based in the frequency that a user used each of them in the interaction processes with the platform. The representation of the knowledge is made with an array (each position has the tag representation and his weight (1)).

$U=($ Tagn (rep, weight); Tagn-1(rep, weight); Tag1(rep, weight);

The tag recommendation process compares the tags present in the user model with the ones existent in the POI model, producing a result based in the number of co-occurrences and specific weight (2).

AssocUserPOIValue $=\sum_{i=1}^{n}$ WeightTagCoocurrence $(i)$

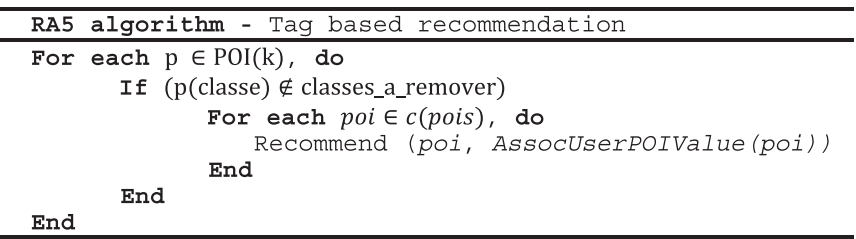

\subsubsection{Social based recommendation model}

Social based recommendation process represents the integration of friends' preferences in the recommendation of each user (this considers that probably exists a similarity between a user preference and his closest friends). SUF1 algorithm is responsible for similarity calculation between a user and his friends. RA6 uses the similarity calculations to produce recommendation results.

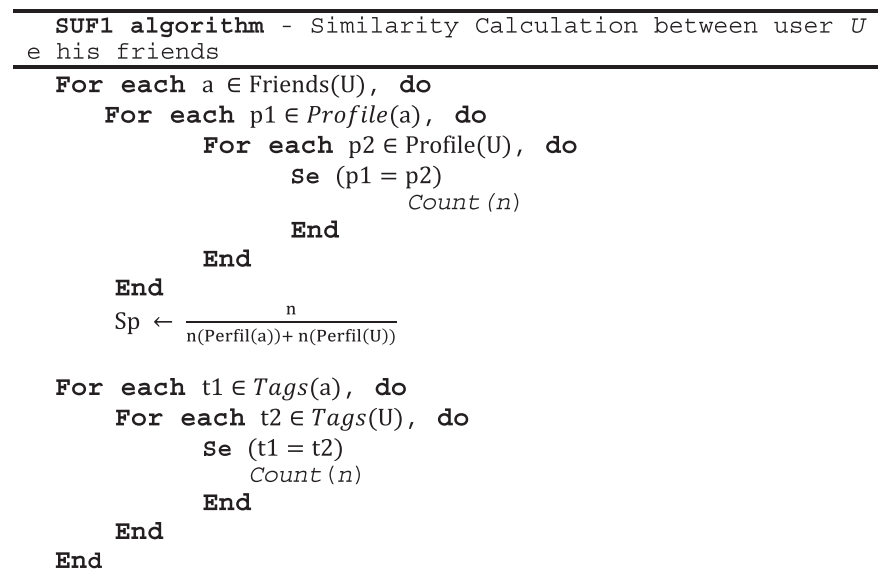

St

$$
\begin{aligned}
& \text { Sf } \leftarrow \frac{S p+S t}{2} \\
& \text { End } \\
& \quad \text { Order }(\text { Friends }(U), S f)
\end{aligned}
$$

The overall recommendation proposal described in this section tries to consider several knowledge sources and filtering technique to enrich the quality of the produced results. The functionality/accessibility model is used to improve the probability of a user fulfils his own ambitions in terms of tourism experiences without being limited by his own physical or psychological conditions.

\section{Prototype}

The architecture proposed in Section 4 was implemented and tested recurring to a web platform developed with this purpose (implemented using PHP and JAVASCRIPT languages). The main functionalities of this platform are a collection of forms and tools (for instance to acquire emotional this prototype implemented a tool called CLMtrackr (Øygard) that allows to understand the user automated response to a stimulus recurring to camera acquired user images) designed to gather personal information about users and POIs and the algorithms (in this prototype were implemented functionality/accessibility, stereotypes and emotional models) described in the section above that are used to create recommendation results.

To test and evaluate this proposal a convenience sample composed by people with various physical and/or psychological handicaps was defined. The criteria to select individuals was that they should have at least carry one the pathologies considered in this work.

In order to test the previously presented artefacts and the developed prototype, the chosen target group was required to fill a set of surveys, aimed at collecting individual user data regarding the models that were implemented in this prototype (functionality/accessibility (RA2), stereotypes (RA3) and emotions (RA4)). In parallel, the research team also collected data from a predefined set of POI introduced all the collected data to the platform, hence allowing it to classify of each of these. In the next phase, the reasoning methods and more specifically the proposed recommendation algorithms were used to produce results.

In this phase each algorithm (RA2, RA3 and RA4) produces its own local recommendation result that will be then used by RA1 algorithm to create the final recommendation results (first RA1 compiles stereotypes (RA3) and emotions (RA4) results recurring to a weighed sum and then this compilation is refined with functionality/accessibility (RA2) results like it is described in Section 4.2.2 above).

Lastly, the final step was the validation of the prototype outputs by performing a set of surveys where the target group was asked to give its opinion on the usability, acceptance, and satisfaction of the prototype recommendations.

\section{Demonstration and tests}

To demonstrate the operation of the proposed artefact, a test scenario using a sample composed by 12 users was built. These users were selected essentially according to their physical or cognitive limitations, hence representing different functionality levels.

The recommendation process begins with the generation of local recommendations in each sub process considered, referring to functionality/ accessibility, stereotypes, emotions, tags and society items. In this particular demonstration only functionality/accessibility, stereotypes and emotions processes were used. The tag and society models were not included

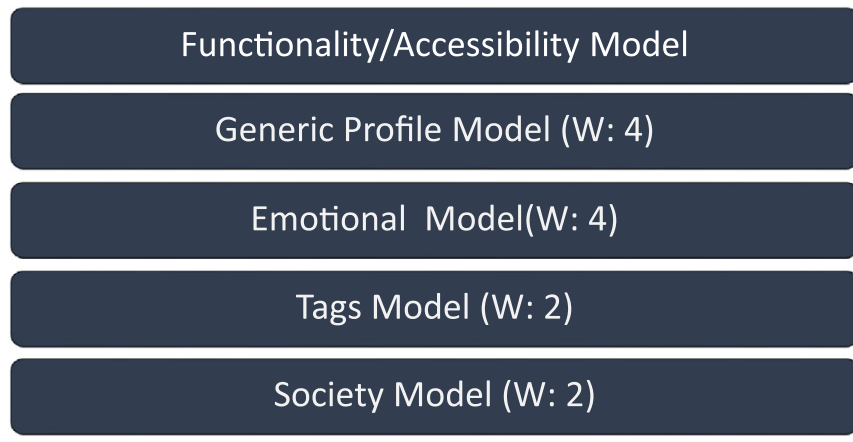

Fig. 5. Hybrid recommendation system components. 
Table 1

Weighted recommendation between Stereotypes and Emotional local results.

\begin{tabular}{llllll}
\hline Users/POI & Pos1 & Pos2 & Pos3 & Pos4 & Pos5 \\
\hline User 1 & POI4 & POI3 & POI1 & POI5 & POI2 \\
User 2 & POI2 & POI5 & POI1 & POI4 & POI3 \\
User 3 & POI2 & POI5 & POI1 & POI4 & POI3 \\
User 4 & POI4 & POI2 & POI3 & POI5 & POI1 \\
User 5 & POI4 & POI2 & POI3 & POI5 & POI1 \\
User 6 & POI2 & POI5 & POI1 & POI4 & POI3 \\
User 7 & POI4 & POI2 & POI5 & POI1 & POI3 \\
User 8 & POI4 & POI5 & POI2 & POI3 & POI1 \\
User 9 & POI2 & POI4 & POI5 & POI1 & POI3 \\
User 10 & POI2 & POI5 & POI1 & POI4 & POI3 \\
User 11 & POI4 & POI3 & POI2 & POI5 & POI1 \\
User 12 & POI2 & POI4 & POI5 & POI1 & POI3 \\
\hline
\end{tabular}

given the lack of existing data to implement the necessary models and ensure their alignment with the test scenario.

The first steps for this process were obtaining the results for both local stereotypes and emotions algorithm. In what concerns the emotional recommendation algorithm, the obtained results were the sum of the emotional reactions obtained for each user/class of POI. The algorithm verifies whether a given POI belongs to a given class, and, if so, considers the perceived reaction to this class as the emotional reaction for this POI.

After obtaining the recommendation values of the local stereotypes and emotions profiling algorithms, it was necessary to generate a recommendation plan that, as previously mentioned is a weighted merge of the local recommendation plans (in this case, each of these models had the value of 4 as defined in Fig. 5). The test resultant plan can be observed in Table 1. Thus, a temporary generic plan was obtained, which was improved by applying these results to the functionality/ accessibility model.

Table 2 is the result of the application of the local functionality/ accessibility algorithm. Each POI position in the results vector is very important, both in the results of functionality/accessibility algorithm and in the results vector of the weighted join between the stereotypes and emotional algorithms (Table 1). POI indexes average in each of these vectors determines the POI indexes in the final vector that will be considered the final recommendation plan (Table 3).

As can be seen in Table 3, among the results produced by the weighted recommendation process (generic profiles and emotions) and the result of final recommendation, there are changes in POI order caused by the combination of the recommendation produced by the functionality/ accessibility model.

The results obtained demonstrate that, for a given user final recommendations vector, POIs worse positioned in the local recommendation vector of the functionality/accessibility are, in most cases, penalized in final recommendation vector (Table 3 ), which allows to guarantee that the POIs that create less obstacles to users, in terms of their own physical or psychological limitations, will be recommended first.

Table 2

Functionality/accessibility local recommendation results.

\begin{tabular}{llllll}
\hline Users/POI & Pos1 & Pos2 & Pos3 & Pos4 & Pos5 \\
\hline User 1 & POI4 & POI3 & POI1 & POI5 & POI2 \\
User 2 & POI2 & POI1 & POI5 & POI4 & POI3 \\
User 3 & POI2 & POI1 & POI5 & POI4 & POI3 \\
User 4 & POI4 & POI2 & POI1 & POI3 & POI5 \\
User 5 & POI3 & POI5 & POI1 & POI4 & POI2 \\
User 6 & POI2 & POI1 & POI4 & POI3 & POI5 \\
User 7 & POI2 & POI1 & POI5 & POI4 & POI3 \\
User 8 & POI1 & POI4 & POI2 & POI3 & POI5 \\
User 9 & POI4 & POI1 & POI3 & POI2 & POI5 \\
User 10 & POI5 & POI1 & POI3 & POI2 & POI4 \\
User 11 & POI4 & POI2 & POI1 & POI3 & POI5 \\
User 12 & POI5 & POI1 & POI3 & POI2 & POI4
\end{tabular}

Table 3

Final improved recommendation results.

\begin{tabular}{llllll}
\hline Users/POI & Pos1 & Pos2 & Pos3 & Pos4 & Pos5 \\
\hline User 1 & POI4 & POI3 & POI1 & POI5 & POI2 \\
User 2 & POI2 & POI1 & POI5 & POI4 & POI3 \\
User 3 & POI2 & POI1 & POI5 & POI4 & POI3 \\
User 4 & POI4 & POI2 & POI3 & POI1 & POI5 \\
User 5 & POI3 & POI4 & POI5 & POI2 & POI1 \\
User 6 & POI2 & POI1 & POI4 & POI5 & POI3 \\
User 7 & POI2 & POI4 & POI1 & POI5 & POI3 \\
User 8 & POI4 & POI1 & POI2 & POI5 & POI3 \\
User 9 & POI4 & POI2 & POI1 & POI3 & POI5 \\
User 10 & POI5 & POI1 & POI2 & POI3 & POI4 \\
User 11 & POI4 & POI2 & POI3 & POI1 & POI5 \\
User 12 & POI5 & POI2 & POI1 & POI4 & POI3 \\
\hline
\end{tabular}

\section{Conclusions and future work}

The main goal and contribution of this research was to show that functionality levels can perform an important role in the user profile creation given that they can be of utmost importance in the definition of what a user can or cannot do, when visiting a specific touristic POI. With this approach, we aimed to prove that this can be the next step in user profile creation, were new information regarding the user's physical and psychological functionality levels, is included. This work also aims to focus the vital importance of classifying POIs according to their own accessibility levels.

The present research also addresses a different perspective in tourism recommendation systems because it includes user's limitations/ differences determined by each individual physical or psychological constraints to accessing touristic locations. This effort is made using an approach where both users and POI's should be characterized regarding their own functionality and accessibility levels in each physical or psychological limitation considered. The correct evaluation/classification of user's functionality and POI accessibility levels is a key factor for the recommendation results produced by the proposed algorithms.

The usage of this kind of approach were users physical and psychological issues are considered by tourism recommendation applications can contribute in practise to allow that more persons with limitations decide to travel because they will gain more trust and confidence in the destination and in themselves.

This research work has a set of limitations namely the ones related with real POI mapping because the information needed to clearly characterize each one implies a huge effort to acquire the information needed. Another limitation of this work is the reduced sample size used in the demonstration process (it was not easy to identify potential tourists with pathologies, available to participate in this study). Sadly, persons with pathologies most of the times already accepted that it is not possible for them to take the same experiences like any other person.

In future developments, we will consider the possibility of defining an ontology to represent the various conceptual domains that can be used to classify POIs which should help in the process of acquiring from several sources already available in a more automated process the information needed to classify them.

To solve the issue related with the reduced sample size and low openness of persons to participate in this kind of study where they it will be fundamental to develop some tools that will be able of acquire information where it is already available (for instance in the several social networks existent). This will help because persons will not be directly questioned which is most of the times considered boring and intrusive.

\section{References}

Abowd, D., Atkeson, G., Hong, J., Long, S., Kooper, R., \& Pinkerton, M. (1997). Cyberguide: A mobile context-aware tour guide. Wireless Networks, 421-433.

Almeida, A., Coelho, B., \& Martins, C. (2010). Intelligent hybrid architecture for tourism services. IFIP advances in information and communication technology, 2010, volume 331, artificial intelligence in theory and practice III. (pp. 205-214), 205-214. 
Ambite, J., Knoblock, C., Muslea, M., \& Minton, S. (2003). Heracles: Hierarchical dynamic constraint networks for interactive planning.

Biuk-Aghai, R. (2003). MacauMap: Tourism-oriented mobile GIS application. Proceedings of map Asia 2003. Kuala Lumpur, Malaysia: GIS Development, 1-8.

Burke, R. (2000). Knowledge-based recommender systems. Encyclopedia of library and information systems. Vol. 69 (32)

Chu, Y., Wang, H., Zheng, L., Wang, Z., \& Tan, K. L. (2016). TRSO: A tourism recommender system based on ontology. In F. Lehner, \& N. Fteimi (Eds.), Knowledge science, engineering and management. KSEM 2016. Lecture notes in computer science, Vol. 9983. Springer.

Coelho, B., Figueiredo, A., \& Martins, C. (2009). Tours planning decision support. ISCIES09. Porto. Portugal.

Colomo-Palacios, R., García-Peñalvo, F. J., Stantchev, V., \& Misra, S. (2017). Towards a social and context-aware mobile recommendation system for tourism. Pervasive and Mobile Computing, 38, 505-515.

Condratov, I. (2013). E-tourism: Concept and evolution. ECOFORUM. Vol. 2, Issue 1(2). (pp. 58) Retrieved from http://www.ecoforumjournal.ro/index.php/eco/article/viewFile/25/20.

Coyle, L., \& Cunningham, P. (2003). Exploiting re-ranking information in a case-based personal travel assistant. Proceedings of the 5th International Conference on case-based reasoning.

DESA, Secretariat for the Convention on the Rights of Persons with Disabilities, Division for Social Policy and Development of the Department of Economic and Social Affairs of the United Nations (DESA) (2016). Accessibility and disability inclusion in urban development. Retrieved from: http://www.un.org/disabilities/documents/ 2016/Urban/DESAissuepaperonAccessibilityandInclusionofPersonswithDisabilitiesin UrbanDevelopment.pdf.

Dimitrios Buhalis, R. L. (2008). Progress in information technology and tourism management: 20 years on and 10 years after the internet-The state of eTourism research tourism management (29), 609-623.

Espinoza, F., Persson, P., Sandin, A., Nyström, H., Cacciatore, E., \& Bylund, M. (2001). GeoNotes: Social and navigational aspects of location-based information systems. In Abowd, Brumitt, \& Shafer (Eds.), Ubicomp 2001: Ubiquitous computing, international conference Atlanta, Georgia, September 30-October 2 (pp. 2-17). Berlin: Springer.

Faria, A., Almeida, A., Martins, C., Gonçalves, R., \& Figueiredo, L. (2015). Personality traits, learning preferences and emotions. Proceedings of the eighth international $C^{*}$ conference on Computer Science \&' Software Engineering (C3S2E '15) (pp. 63-69). New York, NY, USA: ACM.

Gleasure, R., \& Grace, A. (2016). Designing an artefact to help users make intervention decisions about their wellness. Journal of Decision Systems, 25(sup1), 261-273. https://doi.org/10.1080/12460125.2016.1187389.

Gonçalves, R., Martins, J., Branco, F., González Castro, M., Cota, M., \& Barroso, J. (2015). A new concept of 3D DCS interface application for industrial production console operators. Universal Access in the Information Society, 14(3), 399-413. https://doi.org/10. 1007/s10209-014-0368-x.

Gregor, S., \& Hevner, A. (2013). Positioning and presenting design science research for maximum impact. MIS Quarterly, 37(2), 337-355.

Hinze, A., \& Buchanan, G. (2005). Cooperating services in a mobile tourist information system. In R. Meersman (Eds.), Proc. on the move to meaningful internet systems 2005: OTM 2005 workshops, LNCS 3762, 12-13. Berlin: Springer-Verlag.

Höpken, W., Fuchs, M., Zanker, M., Beer, T., Eybl, A., Flores, S., ... Schnabl, M. (2006). etPlanner: An IT framework for comprehensive and integrative travel guidance. Proceedings of ENTER.

UN (2016). http://www.un.org/disabilities/documents/2016/Urban/DESAissuepaperon AccessibilityandInclusionofPersonswithDisabilitiesinUrbanDevelopment.pdf.

Hu, Y., Gao, S., Janowicz, K., Yu, B., Li, W., \& Prasad, S. (2015). Extracting and understanding urban areas of interest using geotagged photos. Computers, Environment and Urban Systems, 54, 240-254. https://doi.org/10.1016/j.compenvurbsys.2015.09.001 (985).

Huang, Y., \& Bian, L. (2009). A Bayesian network and analytic hierarchy process based personalized recommendations for tourist attractions over the internet. Expert Systems with Applications, 36(1), 933-943.

Ibáñez, J., Sebastia, L., \& Onaindia, E. (2016). Planning Tourist Agendas for Different Travel Styles. ECAI, 1818-1823.

Kabassi, K. (2010). Personalizing recommendations for tourists. Telematics and Informatics, 27(1), 51-66. https://doi.org/10.1016/j.tele.2009.05.003.

Kobsa, A. (2001). Generic user modelling systems. User modelling and user-adapted interaction. Kluwer Academic Publishers, 49-63.

Krosche, J., Baldzer, J., \& Boll, S. (2004). MobiDENK-Mobile Multimedia in Monument Conservation. IEEE Multimedia, 11(2), 72-77.

Lucas, J. P., da Silva Coelho, B. E., García, M. N. M., de Almeida Figueiredo, A. M., \& Martins, C. L. (2011). Applying recommender methodologies in tourism sector. Highlights in practical applications of agents and multiagent systems (pp. 101-108). Berlin Heidelberg: Springer.

Luz, N., Anacleto, R., Martins, C., Almeida, A., \& Lucas, J. P. (2013). Lightweight tourism recommendation. International Journal of Web Engineering and Technology, 8(2), 106-123 (9).

Malaka, R., \& Zipf, A. (2000). DEEP MAP ñ challenging IT research in the framework of a tourist information system. In D. Fesenmaier, S. Klein, \& D. Buhalis (Eds.), Information and communication technologies in tourism 2000 (pp. 15-27). New York, Springer: Wien.

Martins, C., Faria, L., De Carvalho, C. V., \& Carrapatoso, E. (2008). User modeling in adaptive hypermedia educational systems. Educational Technology E Society, 11(1), 194-207.

Martins, C., Faria, L., Fernandes, M., Couto, P., Bastos, C., \& Carrapatoso, E. (2013). PCMATmathematics collaborative educational system. Intelligent and adaptive educationallearning systems (pp. 183-212). Berlin Heidelberg: Springer.

Martins, J., Gonçalves, R., Branco, F., Barbosa, L., Melo, M., \& Bessa, M. (2017). A multisensory virtual experience model for thematic tourism: A Port wine tourism application proposal. Journal of Destination Marketing \& Management, 6(2), 103-109. https://doi org/10.1016/j.jdmm.2017.02.002.

McKenzie, G., Janowicz, K., Gao, S., \& Gong, L. (2015, November). How where is when? On the regional variability and resolution of geosocial temporal signatures for points of interest. Computers, Environment and Urban Systems0198-9715, 54, 336-346. https://doi.org/10.1016/j.compenvurbsys.2015.10.002.

McKenzie, G., Janowicz, K., Gao, S., Yang, J. A., \& Hu, Y. (2014). POI pulse: A multi-granular, semantic signatures-based information observatory for the interactive visualization of big geosocial data. Cartographica, 50(2), 71-85.

Michopoulou, E., \& Buhalis, D. (2013). Information provision for challenging markets: The case of the accessibility requiring market in the context of tourism. Information Management, 50(5), 229-239.

Neumann, P. (2002). Barrierefreier Tourismus. Vom Tourismus für Menschen mit Behinderung zum Tourismus für Alle. Germany: Universität Paderborn.

Noguera, J. M., Barranco, M. J., Segura, R. J., \& Martínez, L. (2012). A mobile 3D-GIS hybrid recommender system for tourism. Information Sciences, 215, 37-52.

Øygard, A. (d). http://auduno.com/post/61888277175/fitting-faces.

Parle, E., \& Quigley, A. (2006). Proximo, location-aware collaborative recommender.

Pashtan, A., Blattler, R., Heusser, A., \& Scheuermann, P. (2003). CATIS: A context-aware tourist information system. Proceedings of IMC 2003, Rostock, Germany.

Peffers, K., Tuunanen, T., Rothenberger, M., \& Chatterjee, S. (2007). A design science research methodology for information systems research. Journal of Management Information Systems, 24(3), 45-77. https://doi.org/10.2753/MIS0742-1222240302.

Rivas-Costa, C., Anido-Rifón, L., Fernández-Iglesias, M., Gómez-Carballa, M., ValladaresRodríguez, S., \& Soto-Barreiros, R. (2014). An accessible platform for people with disabilities. International Journal of Human-Computer Interaction, 30(6), 480-494.

Salsgiver, R. (2015). Disability: A diversity model approach in human service practice. Oxford University Press.

Santos, F., de Almeida, A., Martins, C., de Oliveira, P. M., \& Gonçalves, R. (2017). In Á Rocha, A. M. R. Correia, H. Adeli, L. P. Reis, \& S. Costanzo (Eds.), Using functionality/ accessibility levels for personalized POI recommendation. Recent advances in information systems and technologies - volume 1 [WorldCIST'17, Porto Santo Island, Madeira Portugal, April 11-13, 2017], Vol. 569. (pp. 539-548). Springer. https://doi.org/10. 1007/978-3-319-56535-4_55.

Santos, J., \& Almeida, A. (2011). Understanding and evolving tag usefulness in CISTI 2011 doctoral symposium (KMDSS).

Santos, J., \& Almeida, A. (2013). Web 2.0: Tagging Usefulness. In A. Madureira, C. Reis, \& V. Marques (Eds.), Computational intelligence and decision making: Trends and applications (pp. 333-343). Dordrecht: Springer Netherlands.

Tung H. -W., \& Soo, V. -W. (2004). A personalized restaurant recommender agent for mobile e-service. Proceedings of the IEEE international conference on e-technology, e-commerce and e-service (pp. 259-262).

Turismo de Portugal IP. http://www.turismodeportugal.pt.

Tusch, R. (2007). Mobile Multimedia Systems.

Wieringa, R. (2014). What is design science? Design science methodology for information systems and software engineering (pp. 3-11). Springer.

Yang, W. -S., \& Hwang, S. -Y. (2013). iTravel: A recommender system in mobile peer-topeer environment. Journal of Systems and Software, 86, 12-20.

Yoshida, D., Song, X., \& Raghavan, V. (2010). Development of track log and point of interest management system using free and open source software. Applied Geomatics, 2(3), 123-135. 